\title{
Hebbian and Anti-Hebbian Spike-Timing-Dependent Plasticity of Human Cortico-Cortical Connections
}

\author{
Giacomo Koch, ${ }^{1,2}$ Viviana Ponzo, ${ }^{1}$ Francesco Di Lorenzo, ${ }^{1}$ Carlo Caltagirone, ${ }^{1,2}$ and Domenica Veniero ${ }^{1}$ \\ ${ }^{1}$ Non-Invasive Brain Stimulation Unit, Santa Lucia Foundation, Institute for Inpatient Treatment and Scientific Studies, I-00179 Rome, Italy, and \\ ${ }^{2}$ Department of Neuroscience, University of Rome Tor Vergata, I-00133 Rome, Italy
}

Learning of new skills may occur through Hebbian associative changes in the synaptic strength of cortical connections [spike-timingdependent plasticity (STDP)], but how the precise temporal relationship of the presynaptic and postsynaptic inputs determines the STDP effects in humans is poorly understood. We used a novel paired associative stimulation protocol to repeatedly activate the short-latency connection between the posterior parietal cortex and the primary motor cortex (M1) of the left-dominant hemisphere. In different experiments, we systematically varied the temporal relationships between the stimuli and the preferential activation of different M1 neuronal populations by applying transcranial magnetic stimulation over M1 with different coil orientations and in different states of cortical excitability (rest vs muscular contraction). We found evidence for the existence of both Hebbian and anti-Hebbian STDP in human long-range connections. The induction of bidirectional long-term potentiation or depression in M1 depended not only on the relative timing between the stimuli but, crucially, on the stimulation of specific neuronal populations and the activity state of the cortex. Our findings demonstrate that these mechanisms are not fixed but susceptible to rapid adaptations. This sudden transition from anti-Hebbian to Hebbian plasticity likely involves local dynamics of interaction with different populations of postsynaptic neurons.

\section{Introduction}

The temporal proximity of novel encoding stimuli is a key element in determining the formation of a new memory. A classic example is Pavlov's dog, which learned to associate a tone with food when these stimuli were repeatedly associated. These ideas have been formalized by the Hebbian rule, proposing that neurons near-simultaneously activated increase their efficacy if the synapse consistently assists the postsynaptic target neuron to generate action potentials (APs) (Markram et al., 2011). In slice preparations, repeated coincidence of EPSPs and postsynaptic APs results in long-term potentiation (LTP) at both hippocampal and neocortical synapses (Markram et al., 1997; Sjöström et al., 2001), thus satisfying the Hebbian postulate. Typically, LTP is induced when presynaptic activity occurs just before postsynaptic spiking in the target cell (pre-pairing $\rightarrow$ post-pairing). Conversely, long-term depression (LTD) is usually induced when the postsynaptic cell fires before the presynaptic input (post-pairing $\rightarrow$ pre-pairing). This type of plasticity is referred to as spiketiming-dependent plasticity (STDP) (Magee and Johnston, 1997;

Received 0ct. 25, 2012; revised March 6, 2013; accepted March 10, 2013.

Author contributions: G.K. and C.C. designed research;V.P. and F.D.L. performed research; G.K. and D.V. analyzed data; G.K. and D.V. wrote the paper.

This work was supported by Italian Ministry of Health Grants RF08.18, MR08.7, and 09.219 (G.K.). We are grateful to Dr. Silvia Picazio and Dr. Sonia Bonni for their help in data collection. Many thanks to Prof. John Rothwell, Dr. Masashi Hamada, Dr. Binith Cheeran, and Prof. Stefano Ferraina for their constructive comments on a previous version of this manuscript.

The authors declare no competing financial interests.

Correspondence should be addressed to Dr. Giacomo Koch, Laboratory of Clinical and Behavioral Neurology, Santa Lucia Foundation, Institute for Inpatient Treatment and Scientific Studies, Via Ardeatina, 306, I-00179 Rome, Italy. E-mail: g.koch@hsantalucia.it.

DOI:10.1523/JNEUROSCI.4988-12.2013

Copyright $\odot 2013$ the authors $\quad 0270-6474 / 13 / 339725-09 \$ 15.00 / 0$
Markram et al., 2011). However, these temporal rules are not irremovable. Recent findings showed that, at synapses more distal from the soma, the timing requirements for pre-pairing/postpairing shift such that the magnitude, and eventually the sign, of synaptic modifications during STDP at distal synapses are profoundly different from that found at proximal inputs (antiHebbian STDP) (Letzkus et al., 2006; Sjöström and Häusser, 2006).

A main objection is that these complex mechanisms have been investigated extensively in brain slices and do not take in account spontaneous synaptic activity that runs in vivo in humans (Feldman, 2012). Although there is some indirect evidence that STDP is a key factor in governing some mechanisms of associative learning in humans (McMahon and Leopold, 2012), its existence is still matter of debate. Moreover, the experiments performed in brain slices cannot take in account the modules of large-scale connectivity that are crucial in ruling cognition (Varela et al., 2001). Here, by using bifocal transcranial magnetic stimulation (TMS) (Rizzo et al., 2009, 2011; Arai et al., 2011; Buch et al., 2011), we provide novel strong evidence supporting the existence of concomitant antithetic forms of STDP mechanisms in largescale connections in humans. We used a novel paired associative stimulation (PAS) protocol (Stefan et al., 2000) to repeatedly activate the short-latency connection between the posterior parietal cortex (PPC) and the primary motor cortex (M1) of the left-dominant hemisphere (Koch et al., 2007) (see Fig. 1A). In different experiments, we systematically varied (1) the timing of the stimuli between PPC and M1 stimulation, (2) the current direction for M1 stimulation, and (3) the hand muscle activity. The temporal relationship between the stimuli was varied to test for the existence of Hebbian bidirectional plasticity, current di- 
Table 1. TMS parameters used in the different experiments

\begin{tabular}{|c|c|c|c|c|c|c|c|c|c|}
\hline Experiment & Subject no. & Age & M1 RMT & AMT PA & AMT AP & $\begin{array}{l}\text { PPC 90\% } \\
\text { RMT }\end{array}$ & $\begin{array}{l}1 \mathrm{mVFDI} \\
\mathrm{PA}\end{array}$ & $\begin{array}{l}1 \mathrm{mVFDI} \\
\mathrm{AP}\end{array}$ & $\begin{array}{l}1 \mathrm{mV} \\
\text { ADM }\end{array}$ \\
\hline $1 \mathrm{~A}$ & 10 & $26.7 \pm 4.7$ & $36 \pm 2.5$ & & & $32.7 \pm 2.3$ & $47 \pm 4.2$ & & \\
\hline $1 \mathrm{~B}$ & 8 & $27.3 \pm 4.8$ & $38 \pm 5.2$ & & & $33.9 \pm 4.7$ & $45 \pm 5.4$ & & \\
\hline $2 A$ & 10 & $28.3 \pm 1.1$ & $35.2 \pm 6.4$ & $30.3 \pm 6.1$ & $40.9 \pm 6.1$ & $31.9 \pm 5.4$ & $44 \pm 4.2$ & $69 \pm 8.1$ & \\
\hline $2 B$ & 7 & $25.3 \pm 1.8$ & $37.1 \pm 4.4$ & $33.8 \pm 6.5$ & $43.8 \pm 3.2$ & $33.2 \pm 4.1$ & $47 \pm 3.1$ & $67 \pm 7.6$ & \\
\hline $2 C$ & 6 & $25.3 \pm 3.8$ & $39.1 \pm 6.2$ & & & $35.1 \pm 3.3$ & $48 \pm 5.2$ & $63 \pm 8.7$ & \\
\hline $3 \mathrm{~A}$ & 8 & $27.3 \pm 3.2$ & $30.3 \pm 2.5$ & $32 \pm 5.2$ & & $28.8 \pm 0.5$ & $41 \pm 3.0$ & & \\
\hline $3 B$ & 6 & $28.6 \pm 2.9$ & $36.8 \pm 4.8$ & & & $32.9 \pm 4.1$ & $42 \pm 3.6$ & & \\
\hline 4 & 8 & $27.1 \pm 4.7$ & $38 \pm 3.2$ & & & $34.5 \pm 2.9$ & $45 \pm 3.3$ & & $49 \pm 5.2$ \\
\hline $5 A$ & 9 & $25.4 \pm 2.1$ & $37 \pm 6.6$ & $30.1 \pm 5.1$ & & $33.4 \pm 5.9$ & $41 \pm 6.6$ & & \\
\hline $5 B, C$ & 7 & $26.2 \pm 2.3$ & $39 \pm 3.7$ & & & $35.6 \pm 5.9$ & $47 \pm 6.1$ & & \\
\hline
\end{tabular}

TMS parameters used in the different experiments. The values indicate the percentage of maximal stimulator output used in the different experimental conditions (mean \pm SD). For details about each experiment, see Materials and Methods.

rection over M1 was changed to investigate the role of different M1 neuronal populations, and muscular activity (rest vs muscular contraction) was varied to explore the role of different states of M1 cortical excitability. We show that PAS protocols can induce both Hebbian and anti-Hebbian STD-like plasticity that are fully compatible with SPTD as defined at the cellular level. These forms of STDP do not merely depend on the timing of the stimuli but indeed on the dynamics of interaction with specific cortical circuits in the postsynaptic neurons.

\section{Materials and Methods}

In total, 29 healthy volunteers ( 15 males and 14 females; ranging from 21 to 38 years) took part in this study (Table 1). All subjects were righthanded based on the Edinburgh Handedness Inventory (Oldfield, 1971). The experimental procedures used here were approved by the local ethics committee and were performed in accordance with the Declaration of Helsinki. Written informed consent was obtained from all recruited subjects before study initiation.

Experiment 1: PAS between the PPC and the M1 with posterior-anterior orientation. We used bifocal TMS to repeatedly activate the connection between the PPC and M1 of the left-dominant hemisphere (Koch et al., 2007, 2008). In experiment $1 \mathrm{~A}(n=10)$, left PPC TMS preceded or followed the $\mathrm{M}_{\mathrm{PA}}$ (PPC-M1 $1_{\mathrm{PA}} \mathrm{PAS}$ ) stimulation by $5 \mathrm{~ms}$. A third condition in which the interstimulus interval (ISI) varied randomly in the same temporal range was included as a control. We then tracked the time course of the aftereffects of PAS protocol by measuring motor-evoked potentials (MEPs) amplitude as an index of M1 excitability. Notably, we assumed that the inputs from PPC to M1 may discharge the same neurons that are activated by the M1 TMS itself (Koch et al., 2007).

We used a bifocal stimulation technique based on two high-power Magstim 200 machines (Fig. 1A). The magnetic stimuli had a nearly monophasic pulse configuration with a rise time of $\sim 100 \mu \mathrm{s}$, decaying back to zero over $\sim 0.8 \mathrm{~ms}$. To measure the MEPs, electromyographic (EMG) traces were recorded from right first dorsal interosseous (FDI) muscles using 9-mm-diameter, $\mathrm{Ag}-\mathrm{AgCl}$ surface cup electrodes. The active electrode was placed over the belly muscle, whereas the reference electrode was located over the metacarpophalangeal joint of the index finger. Responses were amplified using a Digitimer D360 amplifier through filters set at $20 \mathrm{~Hz}$ and $2 \mathrm{kHz}$ with a sampling rate of $5 \mathrm{kHz}$ and then recorded by a computer using SIGNAL software (Cambridge Electronic Devices). For M1 TMS, the coil was positioned over the hand motor area of left M1, defined as the point at which stimulation evoked the largest MEPs from the contralateral FDI muscle. The stimulator for M1 was connected to a small custom-made figure-of-eight-shaped coil (50 mm external diameter). The intensity of M1 TMS was adjusted to evoke an MEP of $\sim 1 \mathrm{mV}$ peak to peak in the relaxed contralateral FDI muscles. The coil was positioned to induce a posterior-anterior (PA) directed current, being held posterolaterally at an angle of $\sim 45^{\circ}$ to the midline.

To best activate the ipsilateral PPC-M1 connection, the conditioning stimulus (CS) was applied over the left PPC at an intensity of $90 \%$ of the ipsilateral resting motor threshold (RMT) (Fig. 1A). RMT was defined as
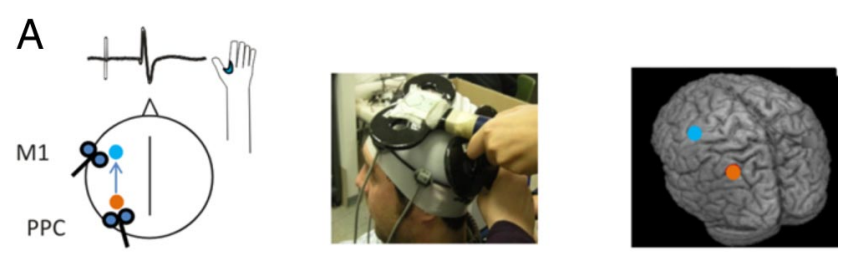

\section{B}

PA
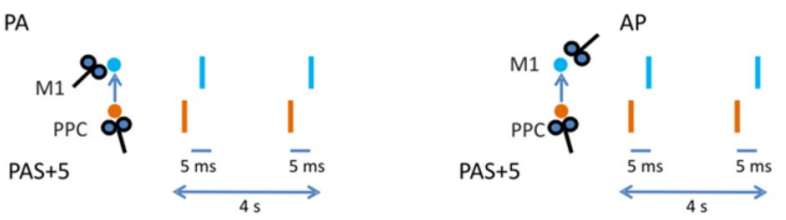

PA
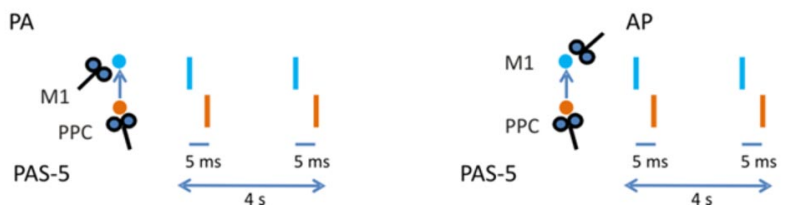

Figure 1. Schematic representation of the experimental procedure. $A$, The PAS protocol was applied over the left PPC and left M1. Mean normalized MNI coordinates of left PPC site were $-48 \pm 4,-65 \pm 3$, and $45 \pm 3 \mathrm{~mm}(x, y, z$, mean $\pm S D)$, and mean MNI coordinates of left M1 were $-30 \pm 3,-12 \pm 3$, and $71 \pm 4(x, y, z$, mean \pm SD). $\boldsymbol{B}$, Across different experiments, we systematically varied the orientation of the stimulating coil over M1 [posterioranterior $\left(M 1_{P A}\right)$ or anterior-posterior $\left(M 1_{A P}\right)$ ] and the ISI for the PPC-M1 PAS. Positive paring indicate that the PPC input precedes the M1 pulse, whereas negative paring indicate that the PPC input is applied after the M1 pulse.

the lowest intensity that evoked five small responses $(\sim 50 \mu \mathrm{V})$ in the contralateral FDI muscle, in a series of 10 stimuli when the subject kept the FDI muscles relaxed in both hands (Rossini et al., 1994). The RMT was assessed at the beginning of each experimental session for each stimulating coil (PPC coil and M1 coil) separately. The magnetic pulse over PPC was applied using a second small custom-made figure-of-eightshaped coil (50 mm external diameter). We used a neuronavigation system (Softaxic; E.M.S.) to precisely position the coil over the PPC sites, using individual T1-weighted magnetic resonance imaging volumes as anatomical reference; this technique has been described previously in detail (Koch and Rothwell, 2009; Koch et al., 2010). The stimulation points were determined before the experiment and were then marked on the adherent plastic cap worn by the subject. The individual coordinates of each stimulation site were then normalized a posteriori into the Montreal Neurological Institute (MNI) coordinate system and averaged (Fig. $1 A$ ). To target PPC, the coil was positioned over the angular gyrus, close to a posterior part of the adjoining caudal intraparietal sulcus. The coordinates of the left PPC were stored and used for neuronavigation in consecutive sessions to ensure comparable stimulation conditions among sessions. The center of the coils was positioned tangentially to the skull with the handle pointing downward and rotated medially by $15^{\circ}$ (Fig. 1A). Indeed, we previously combined TMS with diffusion tensor 
imaging-based tractography to provide evidence for the anatomical bases of the PPC-M1 interaction, revealing that PPC is strongly connected with $\mathrm{M} 1$ by bundles of the superior longitudinal fasciculus (Koch et al., 2010, 2011).

For the PAS protocol, 100 pairs of stimuli were continuously delivered at a rate of $0.2 \mathrm{~Hz}$ for $\sim 8.3 \mathrm{~min}$. The PPC stimulus preceded (PPC$\mathrm{M}_{\mathrm{PA}} \mathrm{PAS}+5 \mathrm{~ms}$ ) or followed (PPC-M1 $1_{\mathrm{PA}} \mathrm{PAS}-5 \mathrm{~ms}$ ) the $\mathrm{M}_{\mathrm{PA}}$ TMS by $5 \mathrm{~ms}$ (Fig. $1 B$ ). In a third control condition (PPC-M1 ${ }_{\mathrm{PA}} \mathrm{PAS}$ Random), the ISI varied randomly for each pair of stimuli in the same temporal range (ISIs: $-5,-4,-3,-2,-1,+1,+2,+3,+4,+5 \mathrm{~ms}$ ). Each subject underwent the three PAS conditions (PPC- $\mathrm{M}_{\mathrm{PA}} \mathrm{PAS}-5 \mathrm{~ms}$, PPC$\mathrm{M} 1_{\mathrm{PA}} \mathrm{PAS}+5 \mathrm{~ms}$, and PPC-M1 $1_{\mathrm{PA}} \mathrm{PAS}$ Random) in 3 different days. These sessions were performed at least 1 week apart. In each session, 20 MEPs were collected and averaged at baseline and $0-5,6-10,11-15$, 16-20, and 21-25 min after PAS. For measuring MEPs, the coil was positioned over the M1 cortical site with the same PA orientation as for the PAS using a standard $7 \mathrm{~cm}$ figure-of-eight coil connected with a Magstim 200 stimulator.

In experiment $1 \mathrm{~B}$, we tested in eight subjects the temporal limits of the PAS interactions. The experimental procedure was the same as experiment 1A, but for the PAS protocol, the PPC preceded or followed the $\mathrm{M} 1_{\mathrm{PA}}$ pulse by 50 or $20 \mathrm{~ms}$ (PPC preceding $\mathrm{M} 1_{\mathrm{PA}}$ TMS: PPC-M1 ${ }_{\mathrm{PA}} \mathrm{PAS}+50 \mathrm{~ms}$, PPC-M1 ${ }_{\mathrm{PA}} \mathrm{PAS}+20 \mathrm{~ms}$; PPC after M1 TMS: $\mathrm{PPC}-\mathrm{M} 1_{\mathrm{PA}} \mathrm{PAS}-50 \mathrm{~ms}, \mathrm{PPC}-\mathrm{M} 1_{\mathrm{PA}} \mathrm{PAS}-20 \mathrm{~ms}$ ). We administered four blocks of stimulation performed in 4 different days. These sessions were performed at least 1 week apart. As in experiment 1, 20 MEPs were collected and averaged at baseline. Then, over the same hotspot, 20 MEPs were recorded at $0-5,6-10,11-15,16-20$, and $21-25 \mathrm{~min}$ after PAS and averaged.

Experiment 2: PAS between the PPC and the M1 with anterior-posterior orientation. In experiment 2 , we tested whether the effects of PAS could change depending on the preferential activation of different neuronal populations, by varying the direction of the induced current over M1 during the PAS protocol. It is known that the neuronal populations activated by TMS in M1 depend on the direction of the induced current in the brain (Day et al., 1989; Di Lazzaro et al., 2006). By simply rotating the stimulating coil over the central sulcus from a PA to an anteriorposterior (AP) direction, it is possible to obtain MEPs with different latencies. This occurs because these different coil orientations activate different set of neurons (Ni et al., 2011), likely related to the activity of the so-called I-waves. These circuits represent synchronous activity of cortico-spinal axons originating from trans-synaptic activation of cortico-spinal cells that occur with different regular delays of $\sim 1.5 \mathrm{~ms}$ (Day et al., 1989; Di Lazzaro et al., 2006) and are known to be mediated by different neuronal populations (Ni et al., 2011).

To confirm that varying the coil orientation would activate MEPs with different latencies in our subjects, we first measured onset latency of MEPs using PA, AP, and lateral-medial (LM) currents during mild contraction of the target muscle $(\sim 10 \%$ of the maximum voluntary contraction). For each orientation (PA, AP, and LM), the hotspot was identified as the position at which the maximum and more stable MEP response was achieved. The hotspot was redefined for each coil orientation. The intensity of stimulation was adjusted relatively on the active motor threshold (AMT), defined as the lowest intensity to evoke an MEP of 200 $\mu \mathrm{V}$ in more than 5 of 10 consecutive trials while subjects maintained $\sim 10 \%$ contraction of the target muscle (Rothwell, 1997). AMT was assessed for each coil orientation. For PA and AP, stimulus intensity was set at $110 \%$ AMT; for LM, it was set at 150\% AMT (Hamada et al., 2012). MEP latencies with different coil orientations were computed by visual inspection in each trial.

We then applied the PAS procedure as in experiment $1 \mathrm{~A}$, but the M1 coil was positioned to induce current in the AP direction (PPC$\mathrm{M} 1_{\mathrm{AP}} \mathrm{PAS}$ ). $\mathrm{M} 1_{\mathrm{AP}}$ TMS was applied over the hotspot of the FDI by rotating the coil handle of $180^{\circ}$ from the PA direction. The intensity of M1 TMS was adjusted to evoke an MEP of $\sim 1 \mathrm{mV}$ peak to peak in the relaxed contralateral FDI muscles. The CS was applied over the left PPC at an intensity of $90 \%$ of the ipsilateral RMT. The PPC stimulus preceded $\left(\mathrm{PPC}-\mathrm{M} 1_{\mathrm{AP}} \mathrm{PAS}+5 \mathrm{~ms}\right)$ or followed (PPC-M1 ${ }_{\mathrm{AP}} \mathrm{PAS}-5 \mathrm{~ms}$ ) the $\mathrm{M} 1_{\mathrm{AP}}$ TMS by $5 \mathrm{~ms}$. Each subject underwent the two PAS conditions in 2 different days, performed at least 1 week apart. Twenty MEPs were collected and averaged at baseline and at $0-5,6-10,11-15,16-20$, and 21-25 min after PAS and averaged.

In the experiment $2 \mathrm{~A}(n=10)$, pre-PAS and post-PAS MEPs measures were collected over M1 with PA current orientation. Then we performed a control experiment [experiment $2 \mathrm{~B}(n=7)$ ] in which MEPs were collected with both PA and AP current orientation. The PPC stimulus preceded (PPC-M1 $1_{\mathrm{AP}} \mathrm{PAS}+5 \mathrm{~ms}$ ) the $\mathrm{M} 1_{\mathrm{AP}}$ TMS by $5 \mathrm{~ms}$.

In experiments $2 \mathrm{~A}$ and $2 \mathrm{~B}$, the coil positioned over $\mathrm{M} 1$ was oriented to induce current in AP direction. This orientation requires higher intensity to be applied to stimulate M1 if compared with the intensity needed with the PA current direction (experiment 1). Such a difference could complicate the interpretation of the results obtained with the two coil orientations because of differences in the magnitude and spatial extent of M1 stimulation rather than differential effects on specific intracortical I-wave circuits.

Thus, we performed another control experiment (experiment $2 \mathrm{C} ; n=$ 6) in which we increased the intensity of M1 stimulation for the PPC$\mathrm{M} 1_{\mathrm{PA}} \mathrm{PAS}$ protocol to make the two experiments comparable in terms of intensity of M1 TMS, regardless of the coil orientation used (AP or PA) (Table 1). We decided to increase M1 TMS in the PPC-M1 ${ }_{\mathrm{PA}} \mathrm{PAS}$ protocol instead of lowering M1 TMS for the PPC-M1 $1_{\mathrm{AP}}$ PAS given that, in this latter case, we would have caused a subthreshold activation of M1 AP circuits and this could have been an additional confound for the comparison between the two experiments. The PPC stimulus preceded (PPC-M1 $1_{\mathrm{PA}} \mathrm{PAS}+5 \mathrm{~ms}$ ) the $\mathrm{M} 1_{\mathrm{PA}}$ TMS by $5 \mathrm{~ms}$.

Experiment 3: state dependency of PAS. In experiment $3 \mathrm{~A}$, performed in a sample of eight subjects, we tested whether the effects of PPC$\mathrm{M} 1_{\mathrm{PA}} \mathrm{PAS}$ protocol could change depending on the state of $\mathrm{M} 1$ excitability. Hence PPC $-\mathrm{M} 1_{\mathrm{PA}} \mathrm{PAS}+5 \mathrm{~ms}$ and $\mathrm{PPC}-\mathrm{M} 1_{\mathrm{PA}} \mathrm{PAS}-5 \mathrm{~ms}$ protocols were applied with subjects performing a mild muscular contraction of the index finger and the thumb finger of the right hand at $10 \%$ of maximal voluntary contraction throughout the entire duration of the PAS protocol. The level of muscular contraction was visually monitored by the experimenter on the basis of the EMG amplitude. $M 1_{\mathrm{PA}}$ TMS was applied over the hotspot of the FDI with intensity sufficient to evoke a 1 $\mathrm{mV}$ MEP as in the experiment 1 . Twenty MEPs were collected and averaged at baseline. Then, 20 MEPs were recorded over the same hotspots at $0-5,6-10,11-15,16-20$, and $21-25$ min after each PAS intervention and averaged.

In experiment $3 \mathrm{~A}$, the intensity of M1 TMS was substantially reduced compared with experiment 1 (Table 1). Therefore, we performed a control experiment in six subjects (experiment $3 \mathrm{~B}$ ) in which PPC-M $1_{\mathrm{PA}} \mathrm{PAS}$ was applied during a mild muscular contraction as in experiment $3 \mathrm{~A}$, but the intensity of M1 TMS was increased to be matched with that used during PPC- $\mathrm{M}_{\mathrm{PA}} \mathrm{PAS}$ at rest (experiment 1 ). The PPC stimulus preceded (PPC-M1 $1_{\mathrm{PA}} \mathrm{PAS}+5 \mathrm{~ms}$ ) the $\mathrm{M}_{\mathrm{PA}}$ TMS by $5 \mathrm{~ms}$.

Experiment 4: topographical M1 specificity of PAS effects. In this experiment, performed in a sample of eight subjects, we tested the effects of $\mathrm{PPC}-\mathrm{M} 1_{\mathrm{PA}} \mathrm{PAS}$ protocols on different hand muscles to investigate whether the effects of the PAS were specific for the stimulated portion of cortical area within M1. MEPs were recorded from surface electrodes over the contralateral abductor digiti minimi (ADM) and FDI muscles on the right hand. We first determined the location of the hotspot for evoking MEPs in the FDI and ADM muscles and marked these points on the scalp. The PPC-M1 $1_{\mathrm{PA}} \mathrm{PAS}+5 \mathrm{~ms}$ protocol was applied, with the $M 1_{\mathrm{PA}}$ TMS delivered over the hotspot of the FDI as in experiment 1. Twenty MEPs were collected and averaged at baseline for each muscle. Then, over the same hotspots, 20 MEPs were recorded at $0-5,6-10$, 11-15, 16-20, and 21-25 min after PAS and averaged.

Experiment 5: effects of PAS on the intracortical circuits of M1. In this experiment, performed in a sample of nine subjects, we tested the effects of PPC-M1 $1_{\mathrm{PA}} \mathrm{PAS}$ protocols (PPC-M1 $1_{\mathrm{PA}} \mathrm{PAS}+5 \mathrm{~ms}$ and PPC$\mathrm{M} 1_{\mathrm{PA}} \mathrm{PAS}-5 \mathrm{~ms}$ ) on the short intracortical inhibition (SICI) and intracortical facilitation (ICF) circuits (experiment 5A). We used a $7 \mathrm{~cm}$ figure-of-eight coil connected with two Magstim 200 stimulators to apply paired TMS over the left motor cortex. The coil was placed at the optimal position for eliciting MEPs from the left FDI muscle. We evaluated the changes occurring in intracortical circuits testing both SICI at 2 
and 3 ms ISIs and ICF at 15 ms ISI (Kujirai et al., 1993) at baseline and after $0-5$ min after PAS. CS was set at $80 \%$ AMT while the intensity of the test stimulus (TS) was adjusted to evoke an MEP of $\sim 1 \mathrm{mV}$ peak to peak in the relaxed FDI. The amplitude of the conditioned MEP at each ISI was expressed as a percentage of the mean peak-to-peak amplitude size of the unconditioned TS in that block. For the PAS protocol, the conditions $\mathrm{PPC}-\mathrm{M} 1_{\mathrm{PA}} \mathrm{PAS}-5 \mathrm{~ms}$ and $\mathrm{PPC}-\mathrm{M} 1_{\mathrm{PA}} \mathrm{PAS}+5 \mathrm{~ms}$ were administered in 2 different days at least 1 week apart.

In experiment $5 \mathrm{~B}$, performed in a sample of seven subjects, we tested the effects of $\mathrm{PPC}-\mathrm{M} 1_{\mathrm{PA}} \mathrm{PAS}$ protocols on the short interval intracortical facilitation (SICF) and the short latency afferent inhibition (SLAI) circuits of M1. We evaluated the changes occurring in SICF and SLAI in two blocks performed at baseline and after PAS. The order of presentation of the blocks was pseudorandomized across subjects. Recordings started 1 min for the first block and 10 min after PAS for the second block. For the PAS protocol, the conditions PPC- $1_{1_{\mathrm{PA}}} \mathrm{PAS}-5 \mathrm{~ms}$ and PPC$\mathrm{M} 1_{\mathrm{PA}} \mathrm{PAS}+5 \mathrm{~ms}$ were administered in 2 different days at least 1 week apart.

For SICF circuit, the TS given alone or the TS followed by the CS at various ISIs were intermixed randomly in one block (Ziemann et al., 1998a). In each block, seven conditions were randomly intermingled: TS alone (MEP) and CS + TS (conditioned MEP for each six different ISIs: $1,1.3,2.1,2.5,3.3$, and $4.1 \mathrm{~ms})$. Before each block, the intensity of TS was adjusted to evoke an MEP of $\sim 1 \mathrm{mV}$ peak to peak in the relaxed left FDI. CS intensity was set at $90 \%$ RMT. Ten responses were collected for paired conditioned MEP for each ISI and for TS alone with a total number of 70 trials in each block. The intertrial interval was set at $5 \mathrm{~s}( \pm 10 \%)$, for a total duration of $\sim 6 \mathrm{~min}$. Measurements were made on each individual trial. The mean peak-to-peak amplitude of the conditioned MEP at each ISI was expressed as a percentage of the mean peak-to-peak amplitude size of the unconditioned test pulse in that block. SLAI measures were studied and recorded using the technique that has been described previously (Sailer et al., 2003). CSs consisted of single pulses $(200 \mu \mathrm{s})$ of electrical stimulation applied to the right median nerve at the wrist through bipolar electrodes (proximal cathode). The intensity of the CS was set at intensity just above motor threshold to evoke a visible twitch of the thenar muscles. The intensity of the cortical magnetic TS was adjusted to evoke a muscle response of $\sim 1 \mathrm{mV}$ peak-to-peak amplitude in the relaxed right FDI. The CS delivered to the peripheral nerve preceded the magnetic TS by different ISIs (16, 20, 24, and $28 \mathrm{~ms})$. Ten responses were collected for each ISI and for TS alone with a total number of 50 trials in each block. The intertrial interval was set to $5 \mathrm{~s}( \pm 10 \%)$, for a total duration of $\sim 5 \mathrm{~min}$. Measurements were collected for each individual trial. The mean peak-to-peak amplitude of the conditioned MEP at each ISI was expressed as a percentage of the mean peak-to-peak amplitude of the unconditioned single-pulse magnetic stimulus in that block.

Data analyses. Data were analyzed using SPSS for Windows version 11.0 on the mean MEPs amplitude in each condition. In experiment $1 \mathrm{~A}$, a two-way repeated-measures ANOVA was performed with protocol $\left(\mathrm{PPC}-\mathrm{M} 1_{\mathrm{PA}} \mathrm{PAS}+5 \mathrm{~ms}\right.$, PPC-M1 $1_{\mathrm{PA}} \mathrm{PAS}-5 \mathrm{~ms}$, and PPC-M1 ${ }_{\mathrm{PA}} \mathrm{PAS}$ Random) and time (baseline and $0-5,6-10,11-15,16-20$, and 21-25 min after PAS) as within-subjects main factors. In experiment $1 \mathrm{~B}$, a two-way repeated-measures ANOVA was performed with protocol $\left(\mathrm{PPC}-\mathrm{M} 1_{\mathrm{PA}} \mathrm{PAS}+50 \mathrm{~ms}, \mathrm{PPC}-\mathrm{M} 1_{\mathrm{PA}} \mathrm{PAS}+20 \mathrm{~ms}, \mathrm{PPC}-\mathrm{M} 1_{\mathrm{PA}} \mathrm{PAS}-20\right.$ $\mathrm{ms}$, and $\mathrm{PPC}-\mathrm{M} 1_{\mathrm{PA}} \mathrm{PAS}-50 \mathrm{~ms}$ ) and time (baseline and $0-5,6-10,11-$ $15,16-20$, and $21-25 \mathrm{~min}$ after PAS) as within-subjects main factors.

In experiment $2 \mathrm{~A}$, a two-way repeated-measures ANOVA was performed with protocol (PPC-M $1_{\mathrm{PA}} \mathrm{PAS}+5 \mathrm{~ms}, \mathrm{PPC}-\mathrm{M} 1_{\mathrm{PA}} \mathrm{PAS}-5 \mathrm{~ms}$, $\mathrm{PPC}-\mathrm{M} 1_{\mathrm{AP}}+5 \mathrm{~ms}, \mathrm{PPC}-\mathrm{M} 1_{\mathrm{AP}}-5 \mathrm{~ms}$ ) and time (baseline and $0-5$, $6-10,11-15,16-20$, and $21-25$ min after PAS) as within-subjects main factors. In experiment $2 \mathrm{~B}$, a two-way repeated-measures ANOVA was performed with coil orientation (PA and AP) and time (baseline and $0-5$, $6-10,11-15,16-20$, and $21-25$ min after PAS) as within-subjects main factors. In experiment $2 \mathrm{C}$, a one-way repeated-measures ANOVA was performed with time (baseline and $0-5,6-10,11-15,16-20$, and 21-25 min after PAS) as within-subjects main factor.

In experiment 3, a two-way repeated-measures ANOVA was performed with protocol (PPC-M1 $1_{\mathrm{PA}} \mathrm{PAS}+5 \mathrm{~ms}$ and $\mathrm{PPC}-\mathrm{M} 1_{\mathrm{PA}} \mathrm{PAS}-5$ $\mathrm{ms}$ ) and time (baseline and $0-5,6-10,11-15,16-20$, and $21-25 \mathrm{~min}$ after PAS) as within-subjects main factors. In experiment 3B, a one-way repeated-measure ANOVA was performed with time (baseline and $0-5$, $6-10,11-15,16-20$, and $21-25$ min after PAS) as within-subjects main factor. In experiment 4, a two-way repeated-measure ANOVA was performed with hand muscle (FDI and ADM) and time (baseline and $0-5$, $6-10,11-15,16-20$, and $21-25 \mathrm{~min}$ after $\left.\mathrm{PPC}-\mathrm{M1}_{\mathrm{PA}} \mathrm{PAS}-5 \mathrm{~ms}\right)$ as within-subjects main factors. In experiment $5 \mathrm{~A}$, a three-way repeatedmeasures ANOVA was performed with protocol ( $\mathrm{PPC}-\mathrm{M} 1_{\mathrm{PA}} \mathrm{PAS}+5 \mathrm{~ms}$ and $\mathrm{PPC}-\mathrm{M} 1_{\mathrm{PA}} \mathrm{PAS}-5 \mathrm{~ms}$ ), time (baseline and $0-5 \mathrm{~min}$ after PAS), and ISI $(2,3$, and $15 \mathrm{~ms})$ as within-subjects main factors. In experiment $5 \mathrm{~B}$, two different three-way repeated-measures ANOVA were performed: one for SLAI protocol with protocol (PPC-M1 ${ }_{\mathrm{PA}} \mathrm{PAS}+5 \mathrm{~ms}$ and PPC$\mathrm{M}_{\mathrm{PA}} \mathrm{PAS}-5 \mathrm{~ms}$ ), time (baseline and after PAS), and ISI (16, 20, 24, and $28 \mathrm{~ms}$ ) as within-subjects main factors for and another for SICF protocol with protocol ( $\mathrm{PPC}-\mathrm{M} 1_{\mathrm{PA}} \mathrm{PAS}+5 \mathrm{~ms}$ and $\mathrm{PPC}-\mathrm{M} 1_{\mathrm{PA}} \mathrm{PAS}-5 \mathrm{~ms}$ ), time (baseline and after PAS), and ISI (1.0, 1.3, 2.1, 2.5, 3.3, and $4.1 \mathrm{~ms}$ ) as within-subjects main factors.

The Greenhouse-Geisser correction was used for nonspherical data. When a significant main effect was reached, paired $t$ tests with Bonferroni's correction were used to characterize the different effects of the specific ISIs. Mauchley's test examined for sphericity. For all statistical analyses, a $p$ value $<0.05$ was considered to be significant.

\section{Results}

In experiment 1A, 100 stimuli of PPC stimulation were paired with $\mathrm{M} 1 \mathrm{TMS}$ with PA orientation (PPC-M1 $1_{\mathrm{PA}}$ ). We found evidence that this PAS protocol induces an anti-Hebbian bidirectional cortico-cortical associative plasticity in the PPC-M1 $1_{\mathrm{PA}}$ network as revealed by the ANOVA showing a main effect of protocol $\left(F_{(2,18)}=9.12 ; p=0.0018\right)$ as well as a protocol $\times$ time interaction $\left(F_{(10,90)}=3.26 ; p=0.0012\right)$. When PPC precedes $\mathrm{M} 1_{\mathrm{PA}}$ stimulation ( $\mathrm{PPC}-\mathrm{M} 1_{\mathrm{PA}} \mathrm{PAS}+5 \mathrm{~ms}$ ), there is a longlasting decrease of the excitability of M1, indicating an LTDlike effect; conversely, when $\mathrm{PPC}$ follows $\mathrm{M} 1_{\mathrm{PA}}$ stimulation (PPC-M $\left.1_{\mathrm{PA}} \mathrm{PAS}-5 \mathrm{~ms}\right)$, there is a long-lasting increase of the excitability of M1, indicating an LTP-like effect. Post hoc analyses showed that for PPC-M $1_{\mathrm{PA}} \mathrm{PAS}+5 \mathrm{~ms}$ there is an inhibition of MEP amplitude compared with baseline at $11-15 \min (p=$ $0.010)$ and $16-20 \mathrm{~min}(p=0.009)$; for $\mathrm{PPC}-\mathrm{M} 1_{\mathrm{PA}} \mathrm{PAS}-5 \mathrm{~ms}$, there is a facilitation of MEP amplitude compared with baseline at $0-5 \min (p=0.010), 6-10 \min (p=0.009), 16-20 \min (p=$ $0.011)$, and $21-25 \mathrm{~min}(p=0.007)$. No significant change is observed when the delay between the two stimuli varies randomly between -5 and +5 ms (Fig. $2 A$ ).

The same effects are still evident when PPC precedes or follows $\mathrm{M} 1_{\mathrm{PA}}$ by $20 \mathrm{~ms}$ but not by $50 \mathrm{~ms}$, as revealed by experiment $1 \mathrm{~B}$ (ANOVA showing a main effect of protocol $\left(F_{(3,21)}=8.30\right.$; $p=0.0007)$, time $\left(F_{(5,35)}=4.45 ; p=0.0030\right)$, as well as a protocol $\times$ time interaction $\left(F_{(15,105)}=3.15 ; p=0.0002\right)$. Post hoc analyses showed that for PPC-M $1_{\mathrm{PA}} \mathrm{PAS}+20 \mathrm{~ms}$ there is an inhibition of MEP amplitude compared with baseline at 6-10 min $(p=0.03)$ and $11-15 \mathrm{~min}(p=0.012)$; for PAS $-20 \mathrm{~ms}$, there is a facilitation of MEP amplitude compared with baseline at $0-5$ $\min (p=0.003), 6-10 \min (p=0.010), 11-15 \min (p=0.008)$, $16-20 \min (p=0.012)$, and $21-25 \min (p=0.011)$ (Fig. $2 B)$. Therefore, experiments $1 \mathrm{~A}$ and $1 \mathrm{~B}$ demonstrate that, in the PPC$\mathrm{M} 1_{\mathrm{PA}}$ network, LTD is obtained when the presynaptic input precedes the postsynaptic activation (positive paring), whereas LTP is evoked in the opposite condition (negative paring), fully satisfying the rules of anti-Hebbian STDP (Fig. 2C). For each experimental condition (PPC-M1 $1_{\mathrm{PA}} \mathrm{PAS}-5 \mathrm{~ms}, \mathrm{PPC}-\mathrm{M} 1_{\mathrm{PA}} \mathrm{PAS}-20$ $\mathrm{ms}, \mathrm{PPC}-\mathrm{M} 1_{\mathrm{PA}} \mathrm{PAS}+5 \mathrm{~ms}$, and PPC-M1 $1_{\mathrm{PA}} \mathrm{PAS}+20 \mathrm{~ms}$ ), we also analyzed eventual changes occurring during the plasticityinducing protocols. In these cases, there were no significant differences in the amplitudes of the MEP conditioned by PPC 

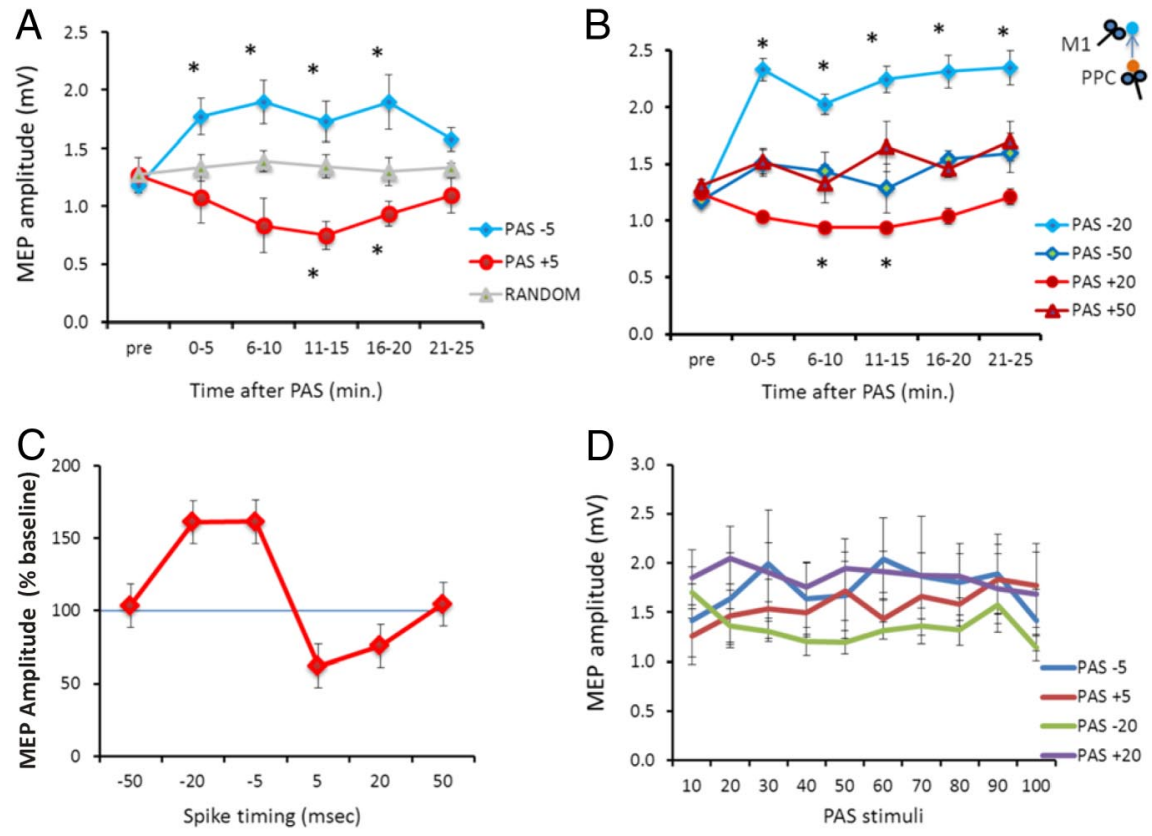

Figure 2. PAS between the $P P C$ and $M 1$ with $P A$ orientation. Aftereffects of $P P C-M 1_{P A}+5 \mathrm{~ms}, P P C-M 1_{P A}-5 \mathrm{~ms}$, and random PAS $(A)$ and PPC $-M 1_{P A}+50 \mathrm{~ms}, P P C-M 1_{P A}+20 \mathrm{~ms}, P P C-M 1_{P A}-20 \mathrm{~ms}$, and PPC $-M 1_{P A}-50 \mathrm{~ms}$ PAS (B) on MEP amplitude at baseline and different time points. $C$, Mean normalized data (percentage of change compared with baseline) obtained at the 11-15 min block after each PAS protocol. The emerging temporal profile follows the rules of anti-Hebbian STDP. D, Mean MEP amplitude during the paired PPC $-\mathrm{M} 1$ plasticity-inducing protocols at $-5,-20,+5$, and $+20 \mathrm{~ms}$. Error bars indicate SEM. ${ }^{*} p<0.05$.

stimulation during the administration of 100 paired stimulations as shown by ANOVA reporting no effects for both the condition $\left(F_{(3,21)}=1.09 ; p=0.37\right)$ and time $\left(F_{(9,63)}=0.76 ; p=0.64\right)$ main factors and for the condition $\times$ time interaction $\left(F_{(27,189)}=0.88\right.$; $p=0.63$ ) (Fig. 2D).

In experiment $2 \mathrm{~A}$, we tested whether the effects of PAS could change depending on the preferential activation of different groups of interneurons. Hence, during PAS administration, we varied the orientation of the stimulating coil over M1 to reverse the direction of induced current from PA to AP direction (PPC$\mathrm{M} 1_{\mathrm{AP}}$ PAS). To verify that this procedure was able to activate different neuronal populations in our sample, we first measured MEP latency at baseline, before any PAS protocol. As expected, we found that different current orientations result in MEPs with different latencies (Fig. 3A). The MEP latencies were $19.5 \pm 1.3$ $\mathrm{ms}$ for $\mathrm{LM}, 20.5 \pm 2.1 \mathrm{~ms}$ for PA, and $23.4 \pm 2.4 \mathrm{~ms}$ for AP current direction (one-way ANOVA main effect, $F_{(2,18)}=50.77$; $p<0.00001$; comparisons between LM and PA, $p<0.01$; between PA and AP, $p<0.001$; between LM and AP, $p<0.001$ ). Notably, the mean conditioned MEP amplitude during corticocortical PAS in this experiment using AP orientation did not differ from that used for PPC-M1 cortico-cortical PAS with PA orientation in experiment $1 \mathrm{~A}$. We then found that the PPC$\mathrm{M} 1_{\mathrm{AP}} \mathrm{PAS}$ protocol induced opposite effects as those obtained in experiment $1 \mathrm{~A}$, as revealed by the ANOVA showing main effects of protocol $\left(F_{(1,9)}=6.81 ; p=0.02\right)$ and time $\left(F_{(5,45)}=3.72 ; p=\right.$ $0.006)$, as well as a protocol $\times$ time interaction $\left(F_{(5,45)}=3.59\right.$; $p=0.008$ ). When PPC precedes $\mathrm{M}_{\mathrm{AP}}$ stimulation (PPC$\left.\mathrm{M} 1_{\mathrm{AP}} \mathrm{PAS}+5 \mathrm{~ms}\right)$, there is a long-lasting increase of the excitability of M1, indicating an LTP-like effect; conversely, when PPC follows $\mathrm{M} 1_{\mathrm{AP}}$ stimulation (PPC-M1 ${ }_{\mathrm{AP}} \mathrm{PAS}-5 \mathrm{~ms}$ ), there is a trend toward a long-lasting decrease of the excitability of M1. Post hoc analyses showed that, for PPC- $\mathrm{M} 1_{\mathrm{AP}} \mathrm{PAS}+5 \mathrm{~ms}$, there is a facilitation of MEPs amplitude compared with baseline at $0-5 \min (p=0.007)$, $6-10 \min (p=0.009), 11-15 \min (p=$ $0.010)$, and $16-20 \mathrm{~min}(p=0.009)$; for $\mathrm{PPC}-\mathrm{M} 1_{\mathrm{AP}} \mathrm{PAS}-5 \mathrm{~ms}$, there is a trend toward a significant inhibition of MEP amplitude compared with baseline at 11-15 $\min (p=0.06)$ (Fig. $3 B)$. Therefore, when we activated different M1 neuronal populations, by changing the direction of the induced current in $\mathrm{M} 1$ ( $\left.\mathrm{PPC}-\mathrm{M} 1_{\mathrm{AP}} \mathrm{PAS}\right)$, we observed an opposite temporal relationship: LTP was evoked when the presynaptic input preceded the postsynaptic activation, whereas for negative timing, there was a trend toward LTD. Hence, STDP followed the temporal rules of classical Hebbian STDP.

In these experiments, although PAS was performed with AP orientation, MEPs were evaluated with the standard PA orientation as in experiments $1 \mathrm{~A}$ and 1B. Therefore, in a control experiment (experiment 2B), we verified whether the PPC-M $1_{\mathrm{AP}} \mathrm{PAS}$ effects would differ if MEPs were tested with different orientations. We found that $\mathrm{PPC}-\mathrm{M} 1_{\mathrm{AP}} \mathrm{PAS}+5$ ms protocol induces similar LTP-like effects in both the AP and PA MEPs conditions as revealed by the ANOVA showing main effects of coil orientation $\left(F_{(1,6)}=12.20 ; p=0.017\right)$ and time $\left(F_{(5,25)}=4.76 ; p=0.003\right)$ but not a coil orientation $\times$ time interaction $\left(F_{(5,25)}=1.14 ; p=0.36\right)$. Post hoc analyses revealed that the LTP-like effects tested with PA MEPs were more durable, showing a facilitation of PA MEP amplitude compared with baseline at $0-5 \min (p=0.007), 6-10 \mathrm{~min}(p=0.009), 11-15 \mathrm{~min}$ $(p=0.010)$, and $16-20 \mathrm{~min}(p=0.009)$. Conversely, the LTPlike effects tested with AP MEPs were shorter, being significant only at $0-5 \min (p=0.04)$ and $6-10 \min (p=0.006)$ (Fig. $3 C)$.

We also analyzed eventual changes occurring during the plasticity-inducing protocols. In these cases, there were no significant differences in the amplitudes of the MEP conditioned by PPC stimulation during the administration of 100 paired stimulations (Fig. 3D).

Experiment 2C showed that the different Hebbian effects obtained in experiments 1 and 2 by changing the orientation of M1 TMS during the PAS protocol cannot be ascribed to differences in the M1 intensity of stimulation. When the M1 TMS in the PPC$\mathrm{M} 1_{\mathrm{PA}} \mathrm{PAS}+5 \mathrm{~ms}$ protocol was increased to be comparable with that used in experiment $2 \mathrm{~A}$, we found the expected LTD-like effect as in experiment 1 (ANOVA showing main effects of time, $F_{(5,25)}=2.72 ; p=0.043$ ) (Table 2).

Experiment $3 \mathrm{~A}$ revealed that the direction of cortical plasticity is also strictly dependent on the M1 state of excitability. When subjects were asked to keep a slight muscular contraction during the PPC-M $1_{\mathrm{PA}} \mathrm{PAS}$ protocol, the effects described in experiment 1 with subjects at rest were fully reversed. The ANOVA showed a main effect of protocol $\left(F_{(1,7)}=11.79 ; p=0.018\right)$ and a protocol $\times$ time interaction $\left(F_{(5,25)}=7.51 ; p=0.002\right)$. Although with subjects at rest PAS $+5 \mathrm{~ms}$ induces LTD-like STDP, during contraction cortical plasticity turns toward LTP, even applying the same PPC-M1 $1_{\mathrm{PA}} \mathrm{PAS}$ protocol. Post hoc analysis showed that, for $\mathrm{PPC}-\mathrm{M} 1_{\mathrm{PA}} \mathrm{PAS}+5 \mathrm{~ms}$, there is a facilitation of MEP amplitude, 
instead of the expected inhibition observed at rest, at $0-5 \min (p=0.010)$, 6-10 $\mathrm{min}(p=0.011)$, and $16-20 \mathrm{~min}$ $(p=0.008)$ compared with baseline (paired $t$ tests). Similarly, for PAS $-5 \mathrm{~ms}$, there is an inhibition of MEP amplitude instead of the expected LTP found at rest at $0-5 \min (p=0.004), 6-10 \min (p=$ $0.002), 11-15 \min (p=0.003)$, and $16-20$ $\min (p=0.0007)$ compared with baseline (Fig. 4). Thus, a transition from antiHebbian to classical Hebbian rules is found when the PPC-M1 $1_{\mathrm{PA}} \mathrm{PAS}$ protocols are applied with subjects performing a slight muscular contraction.

Experiment 3B showed that the different Hebbian effects obtained in experiments 1 and 3 by changing the state of muscular activity during the PAS protocol cannot be ascribed to differences in the M1 intensity of stimulation. When the M1 TMS in the PPC-M $1_{\mathrm{PA}} \mathrm{PAS}+5 \mathrm{~ms}$ protocol with muscular contraction was increased to be comparable with that used in experiment 1, we found the same LTP-like aftereffects as in experiment $3 \mathrm{~A}$ (ANOVA showing main effects of time, $F_{(5,25)}=$ 2.67; $p=0.045)$ (Table 2).

Experiment 4 showed that the aftereffects of PAS are highly specific to the M1 cortical area that is paired with PPC stimulation. In fact, although the LTP effect is observed when PPC-M $1_{\mathrm{PA}}$ PAS occurs between the PPC and the cortical area of the FDI that is stimulated during the PAS protocol, no change is observed for the motor cortical representation of another close hand muscle in $\mathrm{M} 1$, the ADM [ANOVA showing a main effect of muscle $\left(F_{(1,7)}=17.70 ; p=\right.$ $0.008)$, time $\left(F_{(5,25)}=3.65 ; p=0.012\right)$, as well as a muscle $\times$ time interaction $\left(F_{(5,25)}=4.78 ; p=0.003\right)$. Also in this experiment, for PPC-M1 $1_{\mathrm{PA}} \mathrm{PAS}-5 \mathrm{~ms}$, there is a facilitation of MEP amplitude compared with baseline at $0-5 \min (p=0.007), 6-10 \mathrm{~min}$ $(p=0.011), 11-15 \min (p=0.009)$, and $16-20 \min (p=0.012)$ (Fig. 5).

Experiment $5 \mathrm{~A}$ performed with $\mathrm{PPC}-\mathrm{M} 1_{\mathrm{PA}} \mathrm{PAS}$ demonstrated the plastic aftereffects are associated with specific changes in M1 intracortical circuits [ANOVA showing a main effect of ISI $\left(F_{(2,16)}=\right.$ 102.96; $p=0.000001)$ as well as a protocol $\times$ time interaction $\left(F_{(1,8)}=15.32 ; p=0.004\right)$ and a protocol $\times$ time $\times$ ISI interaction $\left.\left(F_{(2,16)}=3.32 ; p=0.041\right)\right]$. Namely, PPC-M1 ${ }_{\mathrm{PA}} \mathrm{PAS}+5 \mathrm{~ms}$ induces a decrease of ICF at $15 \mathrm{~ms}(p=0.002)$, whereas the opposite effect is observed for the PPC-M1 ${ }_{\mathrm{PA}} \mathrm{PAS}-5 \mathrm{~ms}$ protocol ( $\left.p=0.031\right)$, suggesting that long-lasting changes in M1 likely involve the specific set of interneurons that mediate ICF (Fig. 6A,B).

Experiment $5 B$ showed that SLAI and SICF circuits were not modified by the PAS protocols tested. For SLAI measurements ANOVA showed only a main effect of ISI $\left(F_{(3,18)}=15.13 ; p=\right.$ $0.00003)$ but not any other significant main factor or interaction (Fig. $7 A, B$ ). For SICF measurements, ANOVA showed only a main effect of ISI $\left(F_{(5,25)}=7.82 ; p=0.0001\right)$ but not any other significant main factor or interaction (Fig. 7C,D).

\section{Discussion}

We provide here novel strong evidence revealing the existence of both anti-Hebbian and Hebbian STDP in human long-range

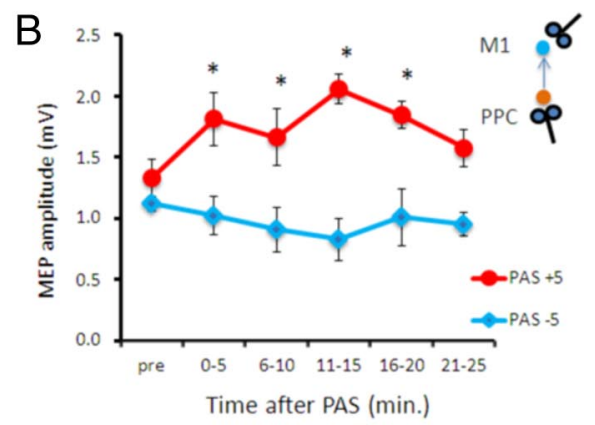

D

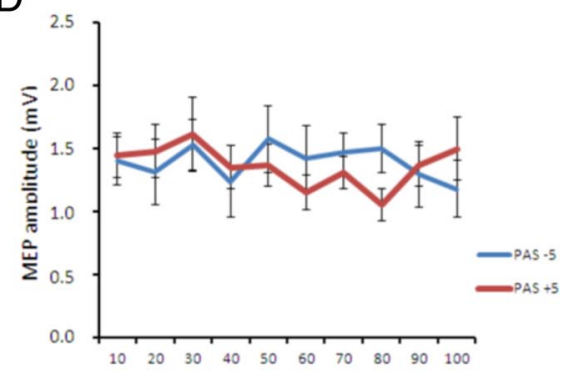

connections. Our findings reinforce the notion that mechanisms of Hebbian-like plasticity can be an important property of cortico-cortical connections (Rizzo et al., 2009; Arai et al., 2011; Buch et al., 2011) and demonstrate that these mechanisms are not fixed but susceptible to rapid adaptations. As discussed below, this sudden transition from anti-Hebbian to Hebbian plasticity likely involves local dynamics of interaction with different populations of postsynaptic neurons.

Our findings reinforce that the opposite Hebbian and antiHebbian STDP effects described here in humans for the first time resemble some recent findings obtained in animal models, describing a switch from Hebbian to anti-Hebbian STDP as the distance of the synaptic contact from the soma increases (Froemke et al., 2005; Sjöström and Häusser, 2006). It was shown that the temporal window of STDP is different at proximal and distal synapses, leading to different degrees of potentiation or depression (Kampa et al., 2007) and that the site of dendritic interaction with the afferent input is a critical element that locally controls the direction of plastic changes within the target cortical neuron (Sjöström and Häusser, 2006). Depending on the relative distance between the site of synaptic interaction with dendritic arbor and the soma of the pyramidal cell, striking different LTP or LTD effects can be induced even when the presynaptic and postsynaptic stimuli are delivered with an identical temporal order. If the presynaptic input precedes the backpropagating APs (bAPs) by few milliseconds (positive timing) and targets dendrites that are localized close to the soma in layer 5, this scheme induces LTP; conversely, if the same experimental procedure is performed targeting the more distal dendrites that are localized in layer $2 / 3$, an opposite LTD phenomenon emerges (Sjöström and Häusser, 2006). Similarly, when the presynaptic input follows the postsynaptic bAP by few milliseconds (negative timing), there is LTP for distal but LTD for proximal inputs (Froemke et al., 2005; Kampa 
Table 2. MEP amplitude for control experiments 2A and 3B

\begin{tabular}{lllllll}
\hline Experiment & Pre & $0-5$ & $6-10$ & $11-15$ & $16-20$ & $21-25$ \\
\hline $2 \mathrm{~A}$ & $1.02 \pm 0.12$ & $1.09 \pm 0.35$ & $1.52 \pm 0.35^{*}$ & $1.24 \pm 0.24^{*}$ & $1.41 \pm 0.35$ & $1.25 \pm 0.24$ \\
$3 \mathrm{C}$ & $1.19 \pm 0.22$ & $1.05 \pm 0.48$ & $0.74 \pm 0.55^{*}$ & $1.07 \pm 0.46$ & $1.15 \pm 0.27$ & $1.29 \pm 0.43$ \\
\hline
\end{tabular}

MEPs amplitude (mean \pm SD) expressed in millivolts. ${ }^{*} p<0.05$ with $t$ test post hoc analysis.

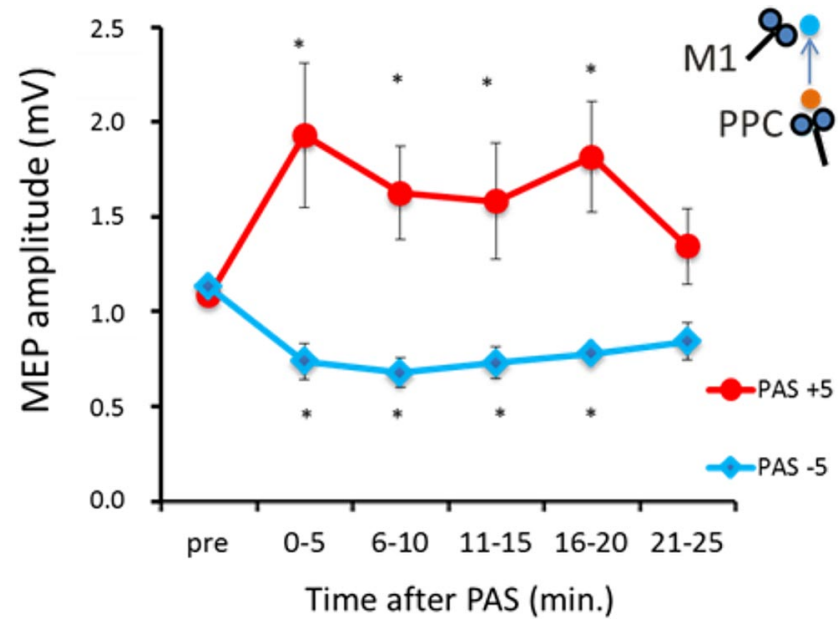

Figure 4. State dependency of PAS. Aftereffects of PPC $-M 1_{P A}+5 \mathrm{~ms}$ and $P P C-M 1_{P A}-5 \mathrm{~ms}$ PAS protocols on MEP amplitude when performed with subjects slightly contracting the FDI muscle during the entire protocol. Note that the effects are opposite to those induced by the same protocols when performed with subjects at rest (see Fig. 2). Error bars indicate SEM. ${ }^{*} p<$ 0.05 .

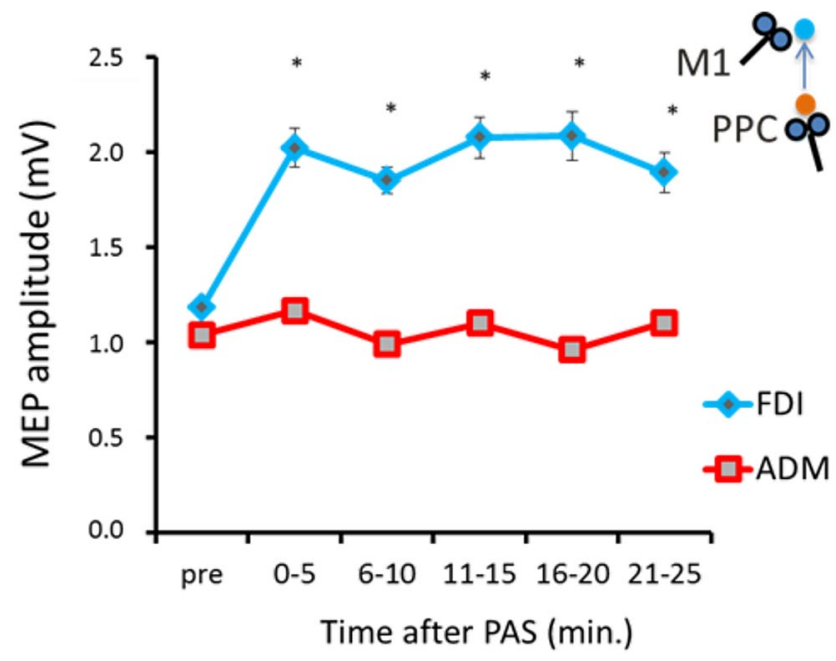

Figure 5. Topographical specificity of PAS effects. Aftereffects of the PPC $-M 1_{P A}-5 \mathrm{~ms} P A S$ protocol on MEP recorded from the FDI and the ADM muscles. The PAS protocol was applied over the "hotspot" area for the FDI. Error bars indicate SEM. ${ }^{*} p<0.05$.

et al., 2007). Notably, the sign of distal plasticity switches from negative (LTD) to positive (LTP) once a sufficient number of distal inputs cooperates, when more inputs are recruited by increasing the stimulation strength (Sjöström and Häusser, 2006).

In our study, the opposite PAS aftereffects observed by varying the orientation of the stimulating coil likely involve the interactions with different M1 neuronal populations. In the M1, TMS with AP and PA orientations is known to induce opposite current flows that activate a set of neurons likely located in different cortical sites (Ni et al., 2011). These opposite AP and PA current flows are supposed to induce upward- or downward-directed

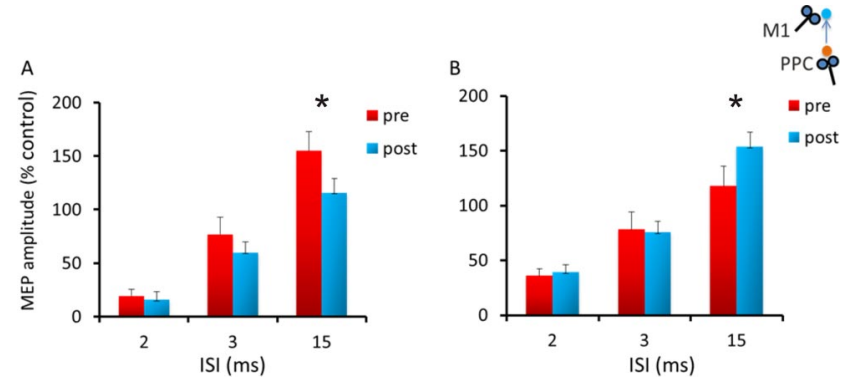

Figure 6. PAS effects on $\mathrm{SICI} / \mathrm{ICF}$ intracortical circuits. Aftereffects of the $\mathrm{PPC}-\mathrm{M} 1_{\mathrm{PA}}+5 \mathrm{~ms}$ $(\boldsymbol{A})$ and PPC $-M 1_{\mathrm{PA}}-5 \mathrm{~ms}(\boldsymbol{B})$ protocols assessed by standard SICl and ICF paired-pulse protocols. Error bars indicate SEM. ${ }^{*} p<0.05$.
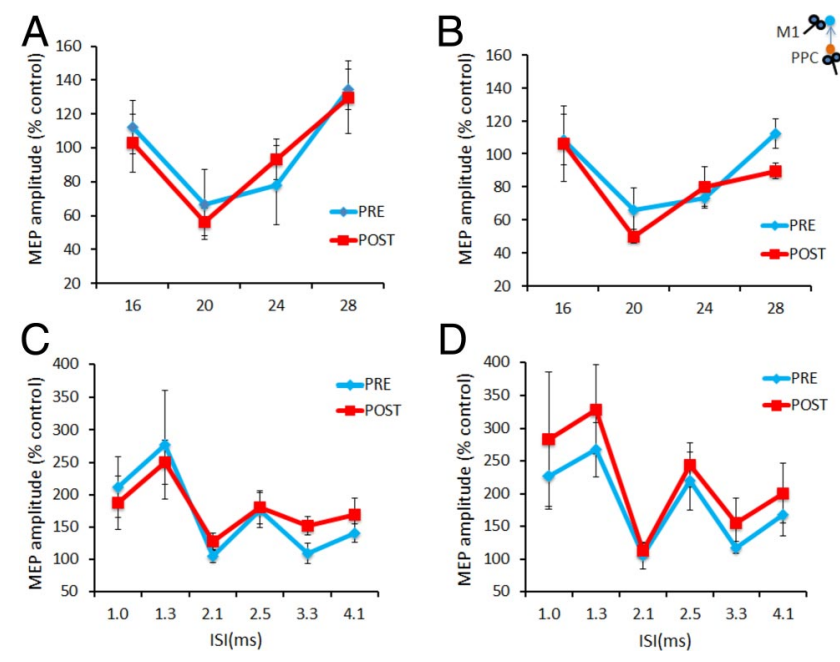

Figure 7. PAS effects on SLAI and SICF intracortical circuits. $A, B$, Aftereffects of the PPC$\mathrm{M1}_{\mathrm{PA}}+5 \mathrm{~ms}(\boldsymbol{A})$ and PPC $-M 1_{P A}-5 \mathrm{~ms}(\boldsymbol{B})$ protocols assessed by SLAl. $\boldsymbol{C}, \boldsymbol{D}$, Aftereffects of the $\mathrm{PPC}-\mathrm{M} 1_{\mathrm{PA}}+5 \mathrm{~ms}(\boldsymbol{C})$ and PPC $-\mathrm{M} 1_{\mathrm{PA}}-5 \mathrm{~ms}(\boldsymbol{D})$ protocols assessed by SICF protocols. Error bars indicate SEM.

current along the dendritic tree of the pyramidal neurons in the anterior bank of the central sulcus (Sommer et al., 2012). It has been suggested that the AP orientation would favor the activation of more apical neuronal populations and be more prone to induce stronger bAPs. In contrast, the PA orientation would induce a current flow directed downward from layer 1 to layer 6 that would be more depolarizing at deeper sites (Sommer et al., 2012). This background could explain why PPC-M $1_{\mathrm{AP}} \mathrm{PAS}$ induces STDP following the temporal rules of classical STDP. AP TMS would more promptly induce stronger bAPs, favoring LTP at more apical layers, in which the PPC inputs arrive (Koch et al., 2007). Conversely, anti-Hebbian STDP would emerge for the PPC-M $1_{\mathrm{PA}}$ PAS because PA TMS provokes weaker distal depolarization, thereby inducing more easily LTD.

It is also possible that these distinct effects could also involve the activity of I-wave interneurons that could mediate the distal afferent input from PPC. According to the canonical model of cortical circuitry, early I-waves are likely to reflect monosynaptic input to cortico-spinal neurons from proximal interneurons, 
whereas more complex oligo-synaptic circuits, including inhibitory interneurons, are involved in late I-wave generation (Day et al., 1989; Sakai et al., 1997; Di Lazzaro et al., 2006, 2012). These interneurons could also have contributed locally to the generation of different forms of STDP. However, the activation of early or late I-wave circuits could have simply caused a temporal shift in the reversal point of the learning rule rather than flipping the sign of the temporal learning rule. Moreover, we did not observe any change in the SICF circuits after PPC-M1 $1_{\mathrm{PA}}$ PAS protocols. Therefore, the possible contribution of the I-wave circuits remains controversial. Another possibility is that AP and PA activated circuits could have involved different synaptic relation with $\mathrm{GABA}_{\mathrm{A}}$ interneurons that have also be invoked in mediating SPTD in humans (Elahi et al., 2012). Therefore, changes in the synaptic efficacy between different neuronal species (pyramidal vs inhibitory neurons) could also produce such effects.

Fascinatingly, the STDP effects were also modulated when the PPC- $\mathrm{M} 1_{\mathrm{PA}} \mathrm{PAS}$ protocols were applied with subjects performing a slight muscular contraction. Also in this case, the direction of induced plasticity flipped completely, now following the timing rules of standard Hebbian STDP. It may well be that, by changing the activation state of $\mathrm{Ml}$ during muscular contraction, the same circuits activated during PPC- $\mathrm{M} 1_{\mathrm{AP}} \mathrm{PAS}$ could become more engaged, leading to a stronger distal dendritic depolarization (Sjöström and Häusser, 2006). The discharge of pyramidal cortical neurons in the course of muscular contraction could increase the coincidence between distally evoked EPSPs with APs satisfying mechanisms of cooperativity among inputs. Interestingly, this state dependency property of cortical plasticity resembles also recent works testing the PAS interactions between other cortical areas with M1 (Arai et al., 2011; Buch et al., 2011) and other protocols, such as theta burst stimulation (Huang et al., 2005; Gentner et al., 2008).

In this regard, it has been suggested that the transition from LTD toward LTP (and vice versa) in these experiments involves the modulation of $\mathrm{Ca}^{2+}$ dynamics at the level of the dendritic synaptic contact. Interestingly, $\mathrm{Ca}^{2+}$ dynamics regulate both the polarity changes of naive synapses exposed to a plasticityinducing stimulation protocol and the physiological events induced by isometric contractions (Wankerl et al., 2010).

Whereas LTP-like plasticity was associated with increased ICF activity, LTD was related with an opposite effect. Although ICF has been related to glutamatergic activity (Ziemann et al., 1998b), the mechanisms of ICF are less clear than those related to SICI. It has been proposed that ICF might result from the recruitment of circuits related to the activation of long-range connections originating from remote areas (Ziemann et al., 1998b). Given that the current PAS protocol is based on the activation of such long-range connections, it is conceivable that the ICF changes could involve the modulation of the same pathways activated by PPC-M1 PAS. Indeed, the specificity of the LTP/LTD-like effect observed in our study was further highlighted by the findings that PPC-M1 PAS induced critical changes only at precise cortical regions within M1. Only MEPs evoked from the motor hotspot stimulated by PAS (recorded from the FDI) were increased after the protocol, whereas no effect at all was found from a close cortical spot in which the excitability of the ADM was tested. These findings resemble the input specificity described in classical PAS protocols. However, the input from PPC likely reaches both the FDI and ADM cortical spots, and we were not able to control for other cortical hotspots that do not receive the cortical projection from PPC.

We did not directly control for any possible spinal effect induced by PPC TMS during the PAS protocols. However, we dem- onstrated previously that PPC TMS at 90\% RMT does not change the amplitude of the $\mathrm{H}$ reflex (Koch et al., 2007), making unlikely this possibility. We also found that the strength of PPC-M1 interactions did not vary in different protocols. Although this might be surprising, this lack of change in the strength of the stimulated conditioning pathway is consistent with classical PAS in which SLAI representing motor cortical inhibition mediated by an ascending central cholinergic projection (Di Lazzaro et al., 2000) does not change (Stefan et al., 2000; Hamada et al., 2012).

The current results obtained with $\mathrm{PPC}-\mathrm{M} 1_{\mathrm{PA}} \mathrm{PAS}$ at rest have an opposite timing to that characterizing the classical median nerve PAS interactions (Stefan et al., 2000). One possibility is that cortico-cortical inputs (mediating the current PPC-M1 interactions) could terminate in different layers than thalamic ones (which mediate the median nerve PAS effects). In fact, thalamocortical neurons synapse on the dendrites of the pyramidal neurons in several cortical layers (McFarland and Haber, 2002). Apart from the more widespread, diffuse terminals to layer 1, there are also strong topographically specific thalamo-cortical projections to deeper layers L2/3 and L5 (McFarland and Haber, 2002).

An intrinsic critique of the current study is that we did not have a direct recording of APs at the postsynaptic neurons, and therefore the current data could be considered as an indirect demonstration of the underlying STDP mechanisms. However, MEPs can be considered as a global measure of cortical excitability, which reflects the summation of thousands of APs. Indeed, the striking analogies between our current findings with the extensive amount of data obtained in animal slices make us very confident in claiming that what we observed were STPD processes occurring at a system level. In particular, our data are compatible with previous animal studies using paired recordings in dissociated neuronal cultures, reporting an $\sim 40$-ms-long coincidence window, with an astoundingly rapid $1 \mathrm{~ms}$ transition between LTP and LTD for near-perfect coincidence between presynaptic and postsynaptic cell activity (Bi and Poo, 1998).

In conclusion, we demonstrate that the time-locked activation of parallel distinct human cortico-cortical connections may lead to antithetic forms of STDP. Additional studies would clarify in the future the behavioral role played by the different forms of Hebbian plasticity described here. For instance, it is possible that these antithetic cortical interactions could play a specific role once new motor memories are encoded or when unnecessary information has to be erased. This interplay could be relevant during processes of adaptation and de-adaptation in visuomotor tasks (Tanaka et al., 2009). From a clinical perspective, the current data could be important to investigate plasticity of specific damaged cortical circuits in patients with motor disorders or to promote novel strategies for stroke-related cortical reorganization (Ridding and Rothwell, 2007; Koch et al., 2012).

\section{References}

Arai N, Müller-Dahlhaus F, Murakami T, Bliem B, Lu MK, Ugawa Y, Ziemann U (2011) State-dependent and timing-dependent bidirectional associative plasticity in the human SMA-M1 network. J Neurosci 31: 15376-15383. CrossRef Medline

Bi GQ, Poo MM (1998) Synaptic modifications in cultured hippocampal neurons: dependence on spike timing, synaptic strength, and postsynaptic cell type. J Neurosci 18:10464-10472. Medline

Buch ER, Johnen VM, Nelissen N, O'Shea J, Rushworth MF (2011) Noninvasive associative plasticity induction in a corticocortical pathway of the human brain. J Neurosci 31:17669-17679. CrossRef Medline

Day BL, Dressler D, Maertens de Noordhout A, Marsden CD, Nakashima K, Rothwell JC, Thompson PD (1989) Electric and magnetic stimulation of 
human motor cortex: surface EMG and single motor unit responses. J Physiol 412:449-473. Medline

Di Lazzaro V, Oliviero A, Profice P, Pennisi MA, Di Giovanni S, Zito G, Tonali P, Rothwell JC (2000) Muscarinic receptor blockade has differential effects on the excitability of intracortical circuits in the human motor cortex. Exp Brain Res 135:455-461. CrossRef Medline

Di Lazzaro V, Pilato F, Oliviero A, Dileone M, Saturno E, Mazzone P, Insola A, Profice P, Ranieri F, Capone F, Tonali PA, Rothwell JC (2006) Origin of facilitation of motor-evoked potentials after paired magnetic stimulation: direct recording of epidural activity in conscious humans. J Neurophysiol 96:1765-1771. CrossRef Medline

Di Lazzaro V, Profice P, Ranieri F, Capone F, Dileone M, Oliviero A, Pilato F (2012) I-wave origin and modulation. Brain Stimul 5:512-525. CrossRef Medline

Elahi B, Gunraj C, Chen R (2012) Short-interval intracortical inhibition blocks long-term potentiation induced by paired associative stimulation. J Neurophysiol 107:1935-1941. CrossRef Medline

Feldman DE (2012) The spike-timing dependence of plasticity. Neuron 75: 556-571. CrossRef Medline

Froemke RC, Poo MM, Dan Y (2005) Spike-timing-dependent synaptic plasticity depends on dendritic location. Nature 434:221-225. CrossRef Medline

Gentner R, Wankerl K, Reinsberger C, Zeller D, Classen J (2008) Depression of human corticospinal excitability induced by magnetic theta-burst stimulation: evidence of rapid polarity-reversing metaplasticity. Cereb Cortex 18:2046-2053. CrossRef Medline

Hamada M, Murase N, Hasan A, Balaratnam M, Rothwell JC (2012) The role of interneuron networks in driving human motor cortical plasticity. Cereb Cortex. Advance online publication. Retrieved March 18, 2013. doi:10.1093/cercor/bhs147. CrossRef Medline

Huang YZ, Edwards MJ, Rounis E, Bhatia KP, Rothwell JC (2005) Theta burst stimulation of the human motor cortex. Neuron 45:201-206. CrossRef Medline

Kampa BM, Letzkus JJ, Stuart GJ (2007) Dendritic mechanisms controlling spike-timing-dependent synaptic plasticity. Trends Neurosci 30: 456-463. CrossRef Medline

Koch G, Rothwell JC (2009) TMS investigations into the task-dependent functional interplay between human posterior parietal and motor cortex. Behav Brain Res 202:147-152. CrossRef Medline

Koch G, Fernandez Del Olmo M, Cheeran B, Ruge D, Schippling S, Caltagirone C, Rothwell JC (2007) Focal stimulation of the posterior parietal cortex increases the excitability of the ipsilateral motor cortex. J Neurosci 27:6815-6822. CrossRef Medline

Koch G, Fernandez Del Olmo M, Cheeran B, Schippling S, Caltagirone C, Driver J, Rothwell JC (2008) Functional interplay between posterior parietal and ipsilateral motor cortex revealed by twin-coil transcranial magnetic stimulation during reach planning toward contralateral space. J Neurosci 28:5944-5953. CrossRef Medline

Koch G, Cercignani M, Pecchioli C, Versace V, Oliveri M, Caltagirone C, Rothwell J, Bozzali M (2010) In vivo definition of parieto-motor connections involved in planning of grasping movements. Neuroimage 51: 300-312. CrossRef Medline

Koch G, Cercignani M, Bonn ì S, Giacobbe V, Bucchi G, Versace V, Caltagirone C, Bozzali M (2011) Asymmetry of parietal interhemispheric connections in humans. J Neurosci 31:8967-8975. CrossRef Medline

Koch G, Bonnì S, Giacobbe V, Bucchi G, Basile B, Lupo F, Versace V, Bozzali $\mathrm{M}$, Caltagirone C (2012) $\theta$-burst stimulation of the left hemisphere accelerates recovery of hemispatial neglect. Neurology 78:24-30. CrossRef Medline

Kujirai T, Caramia MD, Rothwell JC, Day BL, Thompson PD, Ferbert A, Wroe S, Asselman P, Marsden CD (1993) Corticocortical inhibition in human motor cortex. J Physiol 471:501-519. Medline

Letzkus JJ, Kampa BM, Stuart GJ (2006) Learning rules for spike timingdependent plasticity depend on dendritic synapse location. J Neurosci 26:10420-10429. CrossRef Medline

Magee JC, Johnston D (1997) A synaptically controlled, associative signal for Hebbian plasticity in hippocampal neurons. Science 275:209-213. CrossRef Medline

Markram H, Lübke J, Frotscher M, Sakmann B (1997) Regulation of synaptic efficacy by coincidence of postsynaptic APs and EPSPs. Science 275: 213-215. CrossRef Medline
Markram H, Gerstner W, Sjöström PJ (2011) A history of spike-timingdependent plasticity. Front Synaptic Neurosci 3:4. CrossRef Medline

McFarland NR, Haber SN (2002) Thalamic relay nuclei of the basal ganglia form both reciprocal and nonreciprocal cortical connections, linking multiple frontal cortical areas. J Neurosci 22:8117-8132. Medline

McMahon DB, Leopold DA (2012) Stimulus timing-dependent plasticity in high-level vision. Curr Biol 22:332-337. CrossRef Medline

Ni Z, Charab S, Gunraj C, Nelson AJ, Udupa K, Yeh IJ, Chen R (2011) Transcranial magnetic stimulation in different current directions activates separate cortical circuits. J Neurophysiol 105:749-756. CrossRef Medline

Oldfield RC (1971) The assessment and analysis of handedness: the Edinburgh inventory. Neuropsychologia 9:97-113. CrossRef Medline

Ridding MC, Rothwell JC (2007) Is there a future for therapeutic use of transcranial magnetic stimulation? Nat Rev Neurosci 8:559-567. CrossRef Medline

Rizzo V, Siebner HS, Morgante F, Mastroeni C, Girlanda P, Quartarone A (2009) Paired associative stimulation of left and right human motor cortex shapes interhemispheric motor inhibition based on a Hebbian mechanism. Cereb Cortex 19:907-915. CrossRef Medline

Rizzo V, Bove M, Naro A, Tacchino A, Mastroeni C, Avanzino L, Crupi D, Morgante F, Siebner HR, Quartarone A (2011) Associative corticocortical plasticity may affect ipsilateral finger opposition movements. Behav Brain Res 216:433-439. CrossRef Medline

Rossini PM, Barker AT, Berardelli A, Caramia MD, Caruso G, Cracco RQ, Dimitrijević MR, Hallett M, Katayama Y, Lücking CH, Maertens de Noordhout AL, Marsden CD, Murray NM, Rothwell JC, Swash M, Tomberg C (1994) Non-invasive electrical and magnetic stimulation of the brain, spinal cord and roots: basic principles and procedures for routine clinical application. Report of an IFCN committee. Electroencephalogr Clin Neurophysiol 91:79-92. CrossRef Medline

Rothwell JC (1997) Techniques and mechanisms of action of transcranial stimulation of the human motor cortex. J Neurosci Methods 74:113-122. CrossRef Medline

Sakai K, Ugawa Y, Terao Y, Hanajima R, Furubayashi T, Kanazawa I (1997) Preferential activation of different I waves by transcranial magnetic stimulation with a figure-of-eight-shaped coil. Exp Brain Res 113:24-32. CrossRef Medline

Sailer A, Molnar GF, Paradiso G, Gunraj CA, Lang AE, Chen R (2003) Short and long latency afferent inhibition in Parkinson's disease. Brain 126: 1883-1894. CrossRef Medline

Sjöström PJ, Häusser M (2006) A cooperative switch determines the sign of synaptic plasticity in distal dendrites of neocortical pyramidal neurons. Neuron 51:227-238. CrossRef Medline

Sjöström PJ, Turrigiano GG, Nelson SB (2001) Rate, timing, and cooperativity jointly determine cortical synaptic plasticity. Neuron 32: 1149-1164. CrossRef Medline

Sommer M, Norden C, Schmack L, Rothkegel H, Lang N, Paulus W (2012) Opposite optimal current flow directions for induction of neuroplasticity and excitation threshold in the human motor cortex. Brain Stimul. Advance online publication. Retrieved March 18, 2013. doi:10.1016/j.brs.2012.07.003. CrossRef Medline

Stefan K, Kunesch E, Cohen LG, Benecke R, Classen J (2000) Induction of plasticity in the human motor cortex by paired associative stimulation. Brain 123:572-584. CrossRef Medline

Tanaka H, Sejnowski TJ, Krakauer JW (2009) Adaptation to visuomotor rotation through interaction between posterior parietal and motor cortical areas. J Neurophysiol 102:2921-2932. CrossRef Medline

Varela F, Lachaux JP, Rodriguez E, Martinerie J (2001) The brainweb: phase synchronization and large-scale integration. Nat Rev Neurosci 2:229-239. CrossRef Medline

Wankerl K, Weise D, Gentner R, Rumpf JJ, Classen J (2010) L-type voltagegated $\mathrm{Ca}^{2+}$ channels: a single molecular switch for long-term potentiation/ long-term depression-like plasticity and activity-dependent metaplasticity in humans. J Neurosci 30:6197-6204. CrossRef Medline

Ziemann U, Tergau F, Wischer S, Hildebrandt J, Paulus W (1998a) Pharmacological control of facilitatory I-wave interaction in the human motor cortex. A paired transcranial magnetic stimulation study. Electroencephalogr Clin Neurophysiol 109:321-330. CrossRef Medline

Ziemann U, Tergau F, Wassermann EM, Wischer S, Hildebrandt J, Paulus W (1998b) Demonstration of facilitatory I wave interaction in the human 
motor cortex by paired transcranial magnetic stimulation. J Physiol 511: 181-190. CrossRef Medline 